\title{
Neurotoxic Effects of Intrathecal Magnesium Sulphate
}

\author{
Levent Ozdogan 1, Handan Sastim 1, Dilsen Ornek* 1, Aysun Postaci 1, Taner Ayerden ${ }^{1}$, \\ Bayazit Dikmen ${ }^{1}$
}

1. Anaesthesia and Reanimation Department, Ankara Numune Training and Research Hospital, Turkey

Received from Anaesthesia and Reanimation Department, Ankara Numune Training and Research Hospital, Ankara, Turkey.

Submitted on January 3, 2012. Approved on February 27, 2012.

\author{
Keywords: \\ Magnesium Sulphate; \\ Injections, \\ Spinal; \\ Toxic Actions; \\ Microscopy, \\ Electron.
}

\begin{abstract}
Summary
Background and objectives: To assess the potential neurotoxic effects at the ultrastructural level of magnesium sulfate administered intrathecally as a single or multi-dose.

Methods: Our study was conducted with 24 Sprague-Dawley rats that weighed 250-300 g. After a 4-hour fast, the rats were given $10 \mathrm{mg} \cdot \mathrm{kg}^{-1}$ xylazine chloride intraperitoneal and then randomly allocated into three groups. Group I $(n=8)$ received $0.9 \%$ normal saline, Group II $(n=8)$ was given one intrathecal injection of $0.02 \mathrm{~mL}$ of $15 \%$ magnesium sulphate, and Group III $(\mathrm{n}=8)$ was given $0.02 \mathrm{~mL}$ of $15 \%$ magnesium sulphate once a day for seven days. The injections were given within $0.40 \times 50 \mathrm{~mm}$ from the lumbar area. After seven days, the animals were sacrificed under anesthesia with an aortic injection of $10 \%$ formaldehyde and their tissues were fixed. The medulla spinalis was then examined and histopathologically evaluated under an electron microscope. The Kruskal-Wallis test was used for statistical evaluation. A value of $p<.05$ was considered to be statistically significant.

Results: Significant neurodegeneration was detected in rats given single or repeated magnesium sulphate injections compared to the control group. The histopathological evaluation score of this group was also high.

Conclusions: Based on electron microscopic examination, we found that intrathecal magnesium sulphate administration induced neurodegeneration.

(c) 2013 Sociedade Brasileira de Anestesiologia. Published by Elsevier Editora Ltda. All rights reserved.
\end{abstract}

\section{Introduction}

The discovery of opioid receptors within the spinal cord has made drug administration through intrathecal or epidural methods universally used for the treatment of acute or chronic

* Correspondence author: Anaesthesia and Reanimation Department; Ankara Numune Training and Research Hospital Ulku Mahallesi Talatpasa Bulvari No: 5, Altindag, Ankara 06100, Turkey

E-mail: dilsenpinar@yahoo.com pain ${ }^{1}$. Magnesium sulphate, which has been used in preeclampsia and eclampsia as an anticonvulsant for many years, has also been used to treat myocardial infarction, some arrhytmias, asthma, pheochromocytoma, and tetanus. In addition, magnesium sulphate, which is regarded as a natural calcium channel blocker, is a non-competitive antagonist for N-MethylD-Aspartate receptors ${ }^{2}$. Previous studies have proposed that magnesium sulphate has a post-operative analgesic effect when used intravenously ${ }^{3}$. In addition, magnesium sulphate has been previously used in humans and animals intrathecally

ISSN/\$ - see front metter @ 2013 Sociedade Brasileira de Anestesiologia. Published by Elsevier Editora Ltda. All rights reserved. 
for its analgesic and neuroprotective effects ${ }^{4,5}$. However, it is known that permanent neurologic sequels may arise following spinal or epidural administration of the drug ${ }^{6}$.

The aim of this study was to investigate the potential neurotoxic effects of the intrathecal use of magnesium sulphate at the ultrastructural level.

\section{Materials and methods}

This study was conducted in the animal laboratory of the Gülhane Military Medical Faculty with permission from the Ankara University Veterinary Faculty Ethics Committee. Twenty-four Sprague-Dawley rats weighing 250-300 g were included in the study. Rats were fed $20 \%$ proteinaceous chow and provided water ad libitum in accordance with the principles of utilization and supervision of laboratory animals. Prior to the experiment, the animals were maintained under a $12 \mathrm{~h}: 12 \mathrm{~h}$ night/day schedule under optimum conditions of 20-22 ${ }^{\circ} \mathrm{C}$ and $55 \%$ humidity. During the experiments, the same conditions were maintained and rats were separately housed in polycarbonate cages.

After a 4-hour fast, the rats were given an intraperitoneal injection of $100 \mathrm{mg} \cdot \mathrm{kg}^{-1}$ ketamine hydrochloride and $10 \mathrm{mg} . \mathrm{kg}^{-1}$ xylazine chloride. After the rats were anesthetized, the surgical area was shaven, cleaned, and then subjected to intrathecal implementation within a $0.40 \times 50 \mathrm{~mm}$ area of the lumbar L5-6 range. After ceretrospinal fluid (CSF) was observed, a total of $0.02 \mathrm{~mL}$ of study solution was injected through a Hamilton injector ( 28 gauge, sharp pointed, SGE, Australia). Following the intrathecal implementation, rats were observed for clinical toxicity until they began to walk on their own and consume nourishment.

The rats were randomly allocated into three groups for intrathecal injections. Group I $(\mathrm{n}=8)$ was given $0.02 \mathrm{~mL}$ $0.9 \%$ normal saline, Group II $(n=8)$ was given $0.02 \mathrm{~mL} 15 \%$ magnesium sulphate, and Group III $(n=8)$ was given $0.02 \mathrm{~mL}$ magnesium sulphate once a day for seven days. Each injection was given within a region of $0.40 \times 50 \mathrm{~mm}$ from the lumbar area. Following immediate observation of mobility for signs of clinical toxicity, all of the rats were sacrificed under anesthesia on the 8th day with an aortic injection of $10 \%$ formaldehyde; then, their medulla spinalis was analyzed.

Tissue samples $\left(1 \mathrm{~mm}^{3}\right)$ from the lumbar area were incubated for two hours in $2 \%$ glutaraldehyde $(\mathrm{pH} 7.4)$ in phosphate-buffered saline (PBS). At the end of the incubation the tissues were washed three times with PBS and then postfixed in $1 \%$ osmium tetroxide for one hour. The tissue samples were then dehydrated in a series of alcohols. Finally, the tissues were treated with propylene oxide and then mounted as tissue blocks using the Araldite CY212 kit 5x100 g Epoxy resin (Araldite CY212). Half gracilis incisions were made in the blocks, which were polymerized at $56^{\circ} \mathrm{C}$ and stained with toluidine blue. Gracilis muscle was then isolated from areas marked after assessment by light microscopy, stained with uranyl acetate-lead citrate, and then assessed with a Carl Zeiss EM 900 transmission electron microscope (TEM).

\section{Histopathological Changes}

Histopathological changes were scored as follows: normal ultrastructure, 0; degenerative changes in mitochondria where the core content and other organelles were normal, 1; and mitochondrial degeneration with disordered structures in the granullose endoplasmic reticulum (GER), defect in core content, or extracellular edema, 2. A value of 0.5 was also used between findings of 0 and 1 , as well as 1 and 2 .

\section{Statistical Evaluation}

The Kruskal-Wallis test was used to determine significance differences between two groups. When an intergroup difference was detected, the Kruskal-Wallis multiple comparison test was applied, in order to determine the group responsible for the difference. All values of $p<.05$ were considered statistically significant.

\section{Results}

The daily mobility of rats in Group I and III and the mobility of rats in all of the groups during observation at the end of the study were normal. None of the animals were excluded from the study for any reason. There was no statistical difference between the rats in all three groups in terms of weight prior to the study (Table $1 ; p>0.05$ ). The median value obtained from histopathologic examinations was 0 (0-0.5) in the control group, 1 (1-1) in Group II, and 2 (1.5-2) in rats from Group III.

In addition there was a statistically significant difference in toxicity scores between all three groups (Figure 1A; $p<0.001)$. Toxicity was significantly greater in rats given a single dose of magnesium sulphate compared to control rate (Figure $1 \mathrm{~B} ; \mathrm{p}=0.002$ ). In addition, rats given repeated doses of magnesium sulphate had higher toxicities than the control (Figure 1C; $p=0.002$ ). Toxicities were also higher if rats were given repeated doses of magnesium sulphate compared to rats given a single dose (Group II vs. II, respectively; Figure 1D; $p=0.008$ ).

In electron microscopic examination of the medulla spinalis, multipolar neurons with normal structures were observed in the control rats, and the cell nucleus was cycloid, centrally located, and euchromatic. The nucleolus was also conspicuous, and the cell cytoplasm contained endoplasmic reticulum cysternas that were nubile. The electron intensity was dependent on the intensity of ribosome. Mitochondria were found to have normal structures and crista (Figure 2).

In Group II, the electron intensity was dependent on the intensity of the ribosomes. The cell nucleus and nucleolus had normal structures, and granule and endoplasmic reticulum cysternas in the cytoplasm were nubile. These findings were similar to the control group. However, when the mitochondria were analyzed, widespread degeneration was observed, and distention and crystallization of the mitochondria were wide-spread (Figure 3).

In Group III, very distinct degenerative changes were observed. Chromatin loss was evident in the nucleus which was not observed. A decrease in the cytoplasmic density was observed, and conspicuous dilatation was detected in endoplasmic reticulum cysternas. Crystalization in different forms was seen in the mitochondria, and very significant degenerative changes were observed in the neurons. The cells observed from Group III also have a unique increase in lysosomes (Figure 4). Based on these observations, ultrastructural neuro-degeneration was concluded in this group. 


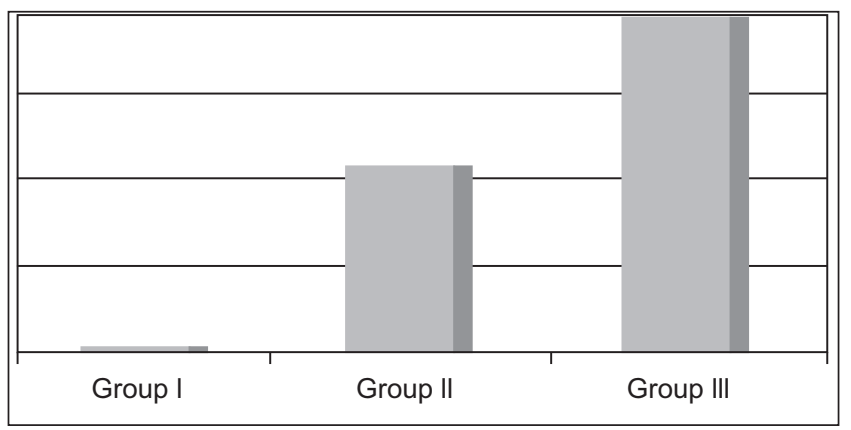

A

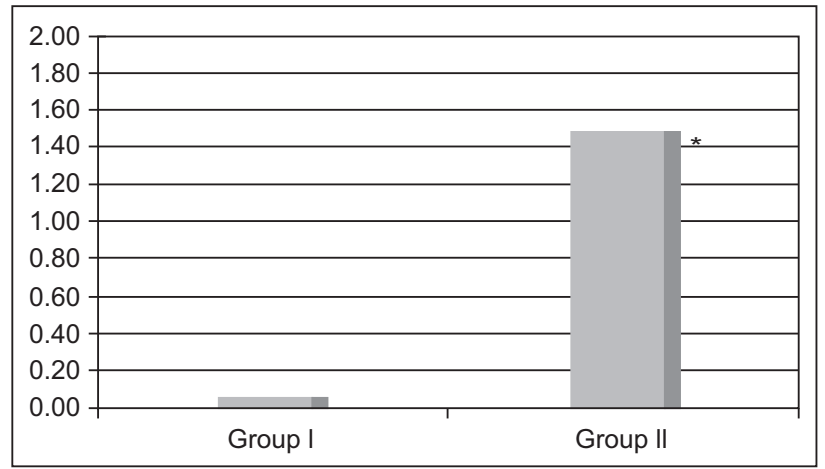

B

Figure 1 A) Toxicity Scores of Group I, Group II, and Group III. B) Comparison of Toxicity Scores of Group I and Group II. C) Comparison of Toxicity Scores of Group I and Group III. D) Toxicity Scores of Group II and Group III.

Table 1 The Body Weights of Rats Before and After the Study.

\begin{tabular}{lllll}
\hline No. & Group & $\begin{array}{l}\text { Body Weight } \\
\text { First Day }(\mathrm{g})\end{array}$ & $\begin{array}{l}\text { Body Weight } \\
\text { Last Day (g) }\end{array}$ & $\mathrm{p}$ \\
\hline I & Control & $268 \pm 17$ & $269 \pm 14$ & $>0.05$ \\
II & Single Dose & $271 \pm 16$ & $273 \pm 15$ & $>0.05$ \\
III & Repeated Dose & $265 \pm 17$ & $266 \pm 15$ & $>0.05$
\end{tabular}

\section{Discussion}

The lack of severe side effects during intravenous usage and the increased application for analgesia indications has made the use of intravenous magnesium sulphate a common subject of scientific studies. More recently, the use of magnesium sulphate has been explored in general anesthesia as well ${ }^{7,8}$. Magnesium sulphate is known to be an N-methyl-D-aspartate (NDMA) receptor antagonist and can block ion channels associated with it. NMDA receptor antagonists may block the central sensitivity associated with peripheral nociceptive stimulation. Studies show that NMDA receptor antagonists have little effect on direct $C$ pain fibrils, but their effect may increase substantially during repeated stimulation ${ }^{9}$. A study by Mitani et al. demonstrated that functional changes in NMDA receptor channels may play specific roles in neuronal damage ${ }^{10}$. Previous studies show that magnesium can reduce acetylcholine release ${ }^{11}$. Since magnesium sulphate depresses cholinergic tonus, this mechanism may play a role in motor block formation ${ }^{12}$. On

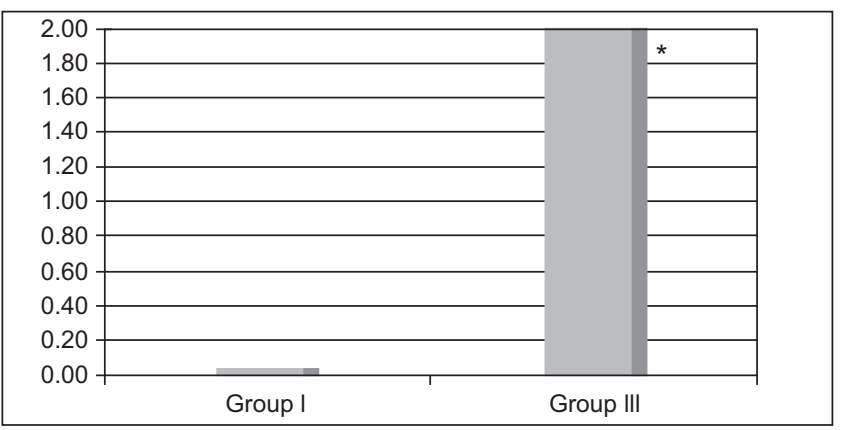

C

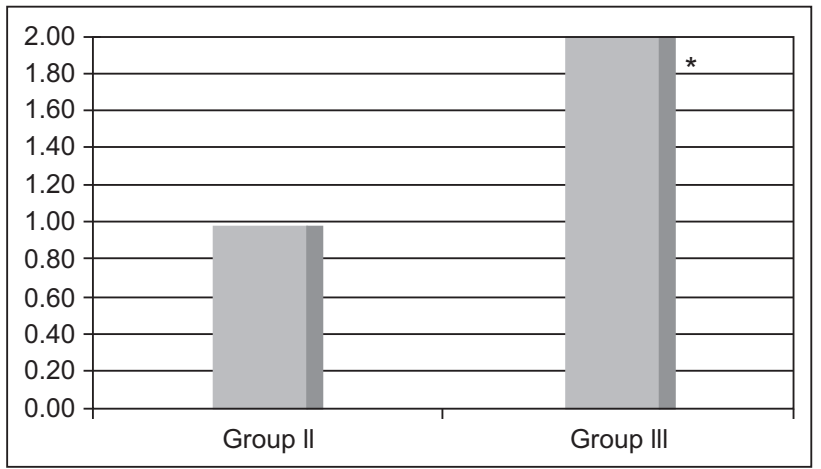

D 


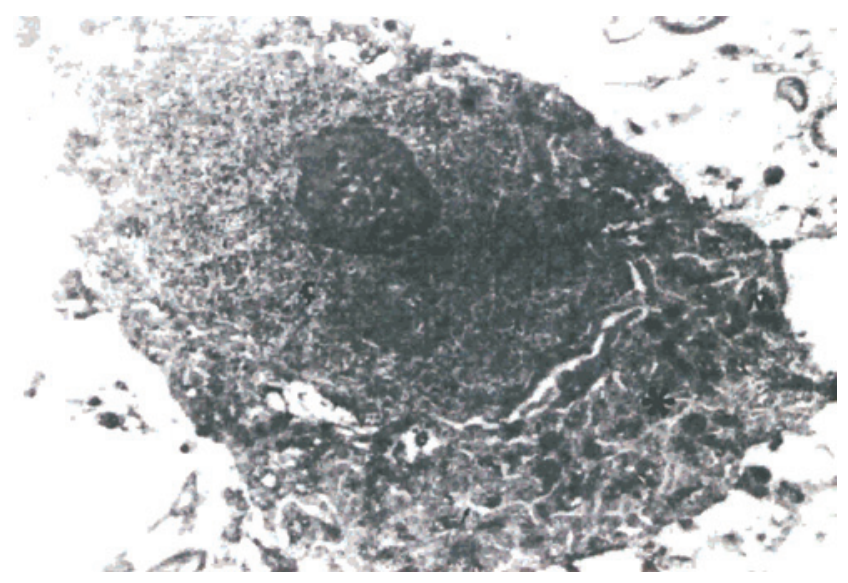

Figure 2 Electron Microscopy of Neurons.

N: Nucleus; GER: Granular Endoplasmic Reticulum; M: Mitochondria; *electron intensive cytoplasm. Examined in normal view (uranyl acetate-lead citrate $440 \times 2.10 \mu \mathrm{m})$.

Nerve lesions are formed after ischemic, traumatic, or toxic effects. These lesions may involve spinal toxicity axons and many components of the neural system that can directly affect the tunics, such as vascular damage and scar tissue. In addition, some local anesthetics may reduce blood stream flow and have toxic effects ${ }^{15,16}$. Moreover, the neurotoxic influence may not only affect cytoplasmic formation, but may also directly affect the nucleus ${ }^{17}$.

Several previous neurotoxicity studies ${ }^{18-20}$ have explored single and multi-dose injections through an intrathecal or epidural catheter. Since the consent is that the use of intrathecal catheters may also cause histopathological changes, we subjected a study group to a repeated dose regimen as well as a single dose regimen, given that both are used in routine clinical practice. In recent studies,

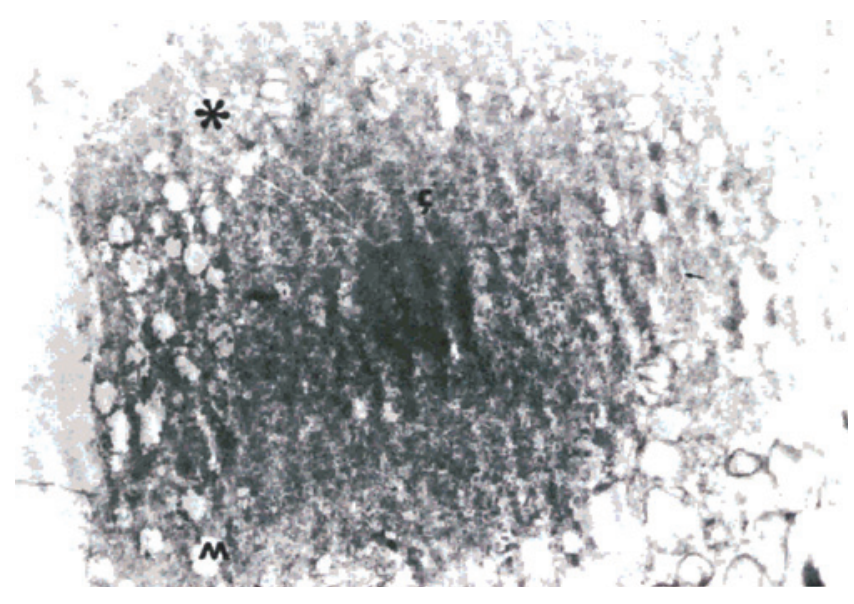

Figure 3 Neuron Electron Microscopic Examination in the Group which was Subjected to Single Dose of Magnesium Sulphate.

N: Nucleus; GER: Granular Endoplasmic Reticulum; *electron intensive cytoplasm. All in normal structure. Crystolysis is observed in mitochondria (uranyl-acetate-lead citrate $440 \times 2.10 \mu \mathrm{m}$ ).

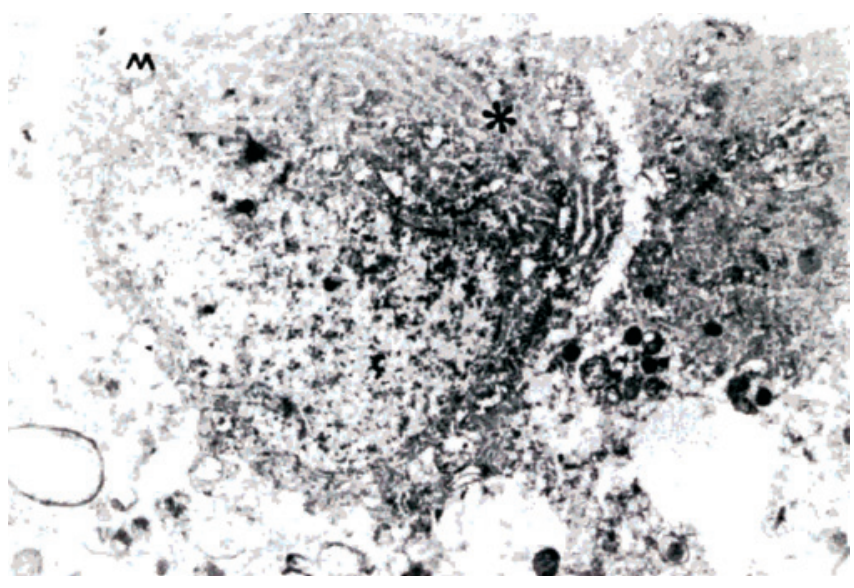

Figure 4 Loss of Chromatin, GER Dilatation, a Decrease in Cytoplasmic Intensity $\left({ }^{*}\right)$ and Crystolysis is conspicuous in the nucleus of the neurons of the group, which was subjected to repeated magnesium sulphate implementation (uranyl acetate-lead citrate $440 \times 2.10 \mu \mathrm{m}$ ).

epidural granulation formation as well as medulla spinalis and massive fibrinolysis in the spinal roots were observed in animal models subjected to intrathecal cannulation and drug administration at epidural and subarachnoid intervals. Parenchymal infarction and abscess were also noted during the infusion of a synthetic drugs. However, since some of these changes were also present in the control group animals, which were only infused with normal saline, it is rather difficult to distinguish the drug-induced changes from other changes due to chronic catheter use alone. In addition, some evidence has indicated that the catheter itself can block the drainage of CSF and causes changes related to this condition ${ }^{21}$.

It is unclear whether morphologic studies used to determine the neurotoxic effects of a compound through intrathecal administration suffice, because the compound-mediated toxicities may have more of an effect on cellular function than cell structure. The absence of morphological changes is not sufficient for determining whether a compound has a potential neurotoxic effect. Therefore, morphological and functional studies must be conducted concomitantly during toxicological analysis of a compound poised for human use ${ }^{22}$.

In this study, significant neurodegeneration was observed after magnesium sulphate administration, particularly during repeated dosing. This degeneration may be a result of an extreme increase in cellular activity and simultaneous deficit in energy metabolism. This degeneration not only affects the cytoplasmic structure, but may also affect the nucleus. Therefore, intracellular magnesium accumulation may also be responsible for neurodegeneration.

Direct neurotoxic effects are thought to be associated with a concentration of previously administered agents ${ }^{23}$. However, in some cases, compounds that have been shown to have potential neurotoxicity in animal models end up being clinically safe in humans at certain doses or concentrations ${ }^{24}$. In this study, we suggest that the intrathecal administration of magnesium sulphate at concentrations of $15 \%$ or higher may cause unforeseen risks for the patient. 
In this study, no disorders in rat motility were observed after the intrathecal administration of magnesium sulphate. Nevertheless, based on the microscopic examination of each group, we hypothesize that the significant neurodegeneration is observed is still important, particularly at the repeated dosing of $15 \%$ magnesium sulphate. Moreover, it is unknown what effect this dosing has on the blood brain barrier, spinal blood stream, and nerve conductions, since the neurophysiological studies could not be implemented due to technical difficulties. In cases where magnesium sulphate administration may be clinically useful, such as for Windup pains that do not respond to opioids ${ }^{25}$, long-term use may be needed. We have not found any neurophysiologic study to date related to the intrathecal usage of magnesium sulphate on experiment animals.

In conclusion, this study suggests that the intrathecal administration of magnesium sulphate at concentrations of $15 \%$ or higher may cause unforeseen risks for the patient. Neurodegeneration was observed by electron microscopy in animals given magnesium sulphate at this concentration, especially after repeated administration, and may be the result of an extreme increase in cellular activity and subsequent deficit in energy metabolism. The neurophysiological studies related to the usage of magnesium sulphate to date have been insufficient for evaluating clinical safety and, therefore, additional studies are needed for full characterization.

\section{References}

1. Barash PG, Culen BF, Stoelting RF - Handbook of Clinical Anesthesia, Management of Acute Post-operative Pain. 3rd edition. Philadelphia. J.B. Lippincott Company 1997; pp. 15471577.

2. Asokumar B, McCarthy RJ, Korin JS Leong W, PerryP, Tuman $\mathrm{KJ}$ - Intrathecal magnesium prolongs fentanyl analgesia. Aneth Analg, 2002;95:661-666.

3. Ko S, Lim H, Kim D, Han Y, Choe H, Song H - Magnesium sulphate does not reduce postoperative analgesic requirements. Anesthesiology, 2001;95:640-646.

4. Dror A, Henriksen E - Accidental epidural magnesium sulphate injection. Anesth Analg, 1987;66:1020-1021.

5. Lejuste $M J$ - Inadvertent intrathecal administration of magnesium of magnesium sulphate. S Afr Med J, 1985;68(6):367-368.

6. Uğur G, Erhan Y, Yegül I - Effects of two different anesthetic medicine on spinal cord and nerve roots. Ege University, Medical Faculty Journal, 1985;24:221-236.

7. Meltzer SJ, Auer J - Physiological and pharmacological studies of magnesium salts. 2. The toxicity of intravenous injections, in particular the effects upon the centers of the medulla. Am J Physiol, 1996;15:387-405.
8. Zalago G, Eisenach JC - Magnesium, anesthesia and hemodynamic control. Anesthesiology, 1991;74:1-10.

9. Bahar M, Berman S, Chanimov M, Weissgarten J, Averbukh $Z$, Cohen ML - Intrathecal anesthesia alters intracellular calcium/magnesium homeostasis in the spinal cord neurons of experimental rats. European Journal of Anesthesiology, 2001;18:231-237.

10. Mitani A, Watanabe M, Kataoka K - Functional change of NMDA receptors related to enhancement of susceptibility to neurotoxicity in the developing pontine nucleus. J Neurosci, 1998;18(19):7941-7952.

11. Fawcett WJ, Haxby EJ, Male DA - Magnesium physiology and pharmacology. 1990;83:302-320.

12. Martyn JA, Standaert FG, Miller RD - Neuromuscular physiology and pharmacology. In: Miller RD (ed). Anesthesia, 5th ed. USA, Churchill Livingstone Inc. 2000; pp. 735-751.

13. Chanimov M, Cohen ML, Grinspun Y, Herbert M, Reif R, Kaufman I, Bahar $M$ - Neurotoxicity after spinal anesthesia induced by serial intrathecal injections of magnesium sulphate. Anaesthesia, 1997;52:223-228.

14. Bahar M, Chanimov M, Grinspun E, Koafman I, Cohen ML - Spinal anaesthesia by intrathecal magnesium sulphate. Anaesthesia, 1996;51:627-633.

15. Diba A - Magnesium sulphate spinal anesthesia. Correspondence. Anaesthesia, 1997;52:187-188.

16. Koinig $\mathrm{H}$, Wallner T, Marhofer $\mathrm{P}$, Andel H, Hörauf K, Mayer N Magnesium sulphate reduces intra and postoperative analgesic requirements. Anesth Analg, 1998;87:206-210.

17. Cheng C, Reynolds IJ - Subcellular localisation of glutamate stimulated intracellular magnesium concentration changes in cultured rat forebrain neurons using confocal microscopy. Neuroscience, 2000;95(4):973-979.

18. Nuutinen L, Laitinen J - A risk-benefit appraisal of injectable NSAID's in the management of postoperative pain. Drug Safety, 1993;9(5):380-393.

19. Ready LB, Plummer MH, Haschke RH - Neurotoxicity of intrathecal local anesthetics in rabbits. Anesthesiology, 1985;63:364-370.

20. Malinovksy JM, Lepage JY, Cozian A, Mussini JM, Pinaudt M - Is ketamine or its preservative responsible for neurotoxicity in the rabbit? Anaesthesiology, 1993;78:109-115.

21. Coombs DW, Fratkin JD. Neurotoxicology of spinal agents. Anesthesiology, 1987;66:724-726.

22. Gordh T Jr, Post C, Olsson Y - Evaluation of the toxicity of subarachnoid clonidine, guanfacine and a substance $P$ antagonist on rat spinal cord and nerve roots. Anesth Analg, 1986;65:13031311.

23. Myers R, Kalichman M, Reisner L, Powell H - Neurotoxicity of local anesthetics. Altered perineural permeability, edema and nerve fiber injury. Anesthesiology, 1986;64:29-35.

24. Rodgson PS, Neal JM, Pollock JE, Liu S - The neurotoxicity of drugs given intrathecally. Anesth Analg, 1999;88:797-809.

25. Pockett S - Spinal cord synaptic plasticity and chronic pain. Anesth Analg, 1995;80:173-179. 